\title{
Slotted-Cylinder Antenna With a Dielectric Coating
}

\author{
James R. Wait and Walter Mientka
}

\begin{abstract}
Analysis is presented for the fields produced by an arbitrary slot on a circular cylinder which has a concentric dielectric coating. Expressions for the far-zone fields are developed by evaluating the appropriate integrals using a saddle-point method. Numerical results are presented for the case of a narrow axial slot for a range of values of cylinder diameters and electrical constants of the dielectric coating. There is some evidence that the coating provides a trap or a duct for surface waves, resulting in an increase of over-all amplitude of the field in the backward direction.
\end{abstract}

\section{Introduction}

Slotted-cylinder antennas are now becoming extensively utilized in microwave radiating systems. The great interest in this subject is evidenced by the great number of papers on this subject within the last decade $[1$ to 7$] .{ }^{1}$ In most of the previous theoretical work, the slot is assumed to be cut on a circular or elliptical cylinder of perfect conductivity and infinite length. The computation of the radiation patterns is then straightforward, although it can be very tedious even if special summation techniques are employed. Usually it is desirable to program the series formula on an electronic calculator if extensive numerical data is required $[8,9]$.

Several years ago one of the authors (J. R. W.) was asked to consider the effect of covering the slot with a dielectric coating such as a fabric. A solution was carried out for an infinitely long axial slot on a circular cylinder, which itself was covered by a concentric dielectric coating of constant thickness [10]. It was shown that, if the coating thickness approached zero, the pattern approached uniformly the pattern expected for the uncoated cylinder. It was then concluded that, if the slot was covered by a lossless dielectric coating of very small thickness, the pattern would not be modified to any extent. Some related experimental work corroborated this conclusion [11].

It is the purpose of the present paper to pursue this matter further. A solution is given for the fields produced by an arbitrary slot on a circular cylinder which is covered by a concentric dielectric coating. Attention is then focused on the special case of an axial slot where the rather cumbersome formulas become less foreboding in appearance. Some numerical results are presented for the far field in the equatorial plane of the slot or in the broadside direction from the cylinder. It is then possible to give a more quantitative viewpoint of the effect of the coating.

It might be mentioned in passing that the plane wave scattering by a dielectric cylinder with a metallic core has been considered recently by Adey

${ }_{1}$ Figures in brackets indicate the literature references at the end of this paper.
[12]. Some of the numerical results obtained by him could have some application to the reciprocal antenna problem for the case when the slot is circumferential.

\section{Formal Solution}

The cylinder is taken to have a radius $a$, and the concentric dielectric coating has a radius $b$, as indicated in figure 1. Cylindrical coordinates $(\rho, \phi, z)$ are chosen to be coaxial with the cylinder. The electrical constants of the coating are $\epsilon$ and $\mu$ and those of the homogeneous (air) space outside are $\epsilon_{0}$ and $\mu_{0}$. The tangential electric fields ${ }^{2}$ on the cylinder are specified, being finite over the area of the slot and zero elsewhere. Therefore, following the suggestion of Silver and Saunders [7], the tangential field is written as a combined azimuthal Fourier series and axial Fourier integral, such that

$$
E_{\phi}(a, \phi, z)=\int_{-\infty}^{\infty} d h \sum_{m=-\infty}^{+\infty} P_{m}(h) e^{-i m \phi} e^{-i h z}
$$

with

$$
P_{m}(h)=\frac{1}{(2 \pi)^{2}} \int_{z_{1}}^{z_{2}} d z^{\prime} \int_{\phi_{2}}^{\phi_{1}} d \phi^{\prime} E_{\phi}\left(a, \phi^{\prime}, z^{\prime}\right) e^{i h z^{\prime}} e^{i m \phi^{\prime}},
$$

where the slot is considered to be bounded by $\phi_{1}<\phi^{\prime}<\phi_{2}$ and $z_{1}<z^{\prime}<z_{2}$ in terms of the primed coordinates. A similar relation holds for the $E_{z}(a, \phi, z)$ component with $P_{m}(h)$ being replaced by $Q_{m}(h)$.

In the subsequent equations, the double Fourier representation is considered as an operator to simplify the notation. For example, eq (1) is rewritten, operationally

$$
E_{\phi}(a, \phi, z)=\Gamma P_{m}(h),
$$

where the $\Gamma$ signifies the multiplication of $P_{m}(h)$ by $\exp [-i h z-i m \phi]$ and then integration with respect to $h$ and summation with respect to $m$.

In region $\mathrm{I}$, defined by $a \leq \rho \leq b$, the fields can be represented as a superposition of TM (transverse magnetic) and TE (transverse electric) modes [13]. 
Therefore, the electric and magnetic field components are given by

$$
E_{z}=\Gamma\left[u^{2} a_{m} H_{m}+u^{2} A_{m} J_{m}\right],
$$

$$
\begin{aligned}
E_{\phi}=\Gamma\left[i \mu \omega b_{m} \frac{\partial H_{m}}{\partial \rho}-\right. & \frac{m h}{\rho} a_{m} H_{m} \\
& \left.\quad+i \mu \omega B_{m} \frac{\partial J_{m}}{\partial \rho}-\frac{m h}{\rho} A_{m} J_{m}\right],
\end{aligned}
$$

$$
\begin{gathered}
E_{\rho}=\Gamma\left[-\frac{m \omega \mu}{\rho} b_{m} H_{m}-i h a_{m} \frac{\partial H_{m}}{\partial \rho}\right. \\
\left.-\frac{m \omega \mu}{\rho} B_{m} J_{m}-i h A \frac{\partial J_{m}}{\partial \rho}\right], \\
H_{z}=\Gamma\left[u^{2} b_{m} H_{m}+u^{2} B_{m} J_{m}\right],
\end{gathered}
$$$$
H_{\phi}=\Gamma\left[-\frac{m h}{\rho} b_{m} H_{m}-\frac{i k^{2}}{\mu \omega} a_{m} \frac{\partial H_{m}}{\partial \rho}\right.
$$$$
\left.-\frac{m h}{\rho} B_{m} J_{m}-\frac{i k^{2}}{\mu \omega} A_{m} \frac{\partial J_{m}}{\partial \rho}\right]
$$

$$
\begin{aligned}
H_{\rho}=\Gamma\left[-i \hbar b_{m} \frac{\partial H_{m}}{\partial \rho}\right. & +\frac{m k^{2}}{\mu \omega \rho} a_{m} H_{m} \\
& \left.\quad-i h B_{m} \frac{\partial J_{m}}{\partial \rho}+\frac{m k^{2}}{\mu \omega \rho} A_{m} J_{m}\right],
\end{aligned}
$$

where $J_{m}=J_{m}(u \rho)$, the Bessel function of the first type of order $m$; where $H_{m}=H_{m}^{(2)}(u \rho)$, the Hankel function of the second kind of order $m$; and where $u=\left(k^{2}-h^{2}\right)^{1 / 2}$ and $k=(\epsilon \mu)^{1 / 2} \omega$. The coefficients $a_{m}$, $b_{m}, A_{m}$, and $B_{m}$ are independent of $\rho, \phi$, and $z$, but are as yet unknown.

In region II, defined by $\rho \geq b$, the representation is also a superposition of TM and TE modes, but now only the Hankel function is needed because it has the proper asymptotic behavior for large values of $\rho$. Therefore,

$$
\begin{gathered}
E_{z}=\Gamma u_{0}^{2} c_{m} H_{m} \\
E_{\phi}=\Gamma\left[i \mu_{0} \omega d_{m} \frac{\partial H_{m}}{\partial \rho}-\frac{m h}{\rho} c_{m} H_{m}\right] \\
E_{\rho}=\Gamma\left[-\frac{m \mu_{0} \omega}{\rho} d_{m} H_{m}-i h c_{m} \frac{\partial H_{m}}{\partial \rho}\right] \\
H_{z}=\Gamma u_{0}^{2} d_{m} H_{m} \\
H_{\phi}=\Gamma\left[-\frac{m h}{\rho} d_{m} H_{m}-\frac{i k_{0}^{2}}{\mu_{0} \omega} c_{m} \frac{\partial H_{m}}{\partial \rho}\right] \\
H_{\rho}=\Gamma\left[-i h d_{m} \frac{\partial H_{m}}{\partial \rho}+\frac{m k_{0}^{2}}{\mu_{0} \omega \rho} c_{m} H_{m}\right],
\end{gathered}
$$

where $H_{m}=H_{m}^{(2)}\left(u_{0} \rho\right), u_{0}=\left(k_{0}^{2}-h^{2}\right)^{1 / 2}$, and $k_{0}=\left(\epsilon_{0} \mu_{0}\right)^{1 / 2} \omega$. The coefficients $c_{m}$ and $d_{m}$ are independent of $\rho, \phi$, and $z$.
The $E_{\phi}$ and $E_{z}$ components in region I must reduce to the prescribed behavior at $\rho=a$, as noted by eq (1). Furthermore, the $E_{\phi}, E_{z}, H_{\phi}$ and $H_{z}$ components are continuous at $\rho=b$. Together these conditions lead to six linear equations to determine the six unknown coefficients. Symbolically, this set is

$a_{m p} a_{m}+b_{m p} b_{m}+A_{m p} A_{m}+B_{m p} B_{m}+c_{m p} c_{m}+d_{m p} d_{m}=x_{m p}$,

where the coefficients with the double suffix are given conveniently in table 1 for $p$ from 1 to 6 . It is now a simple matter to solve for the coefficients in determinant form. For example:

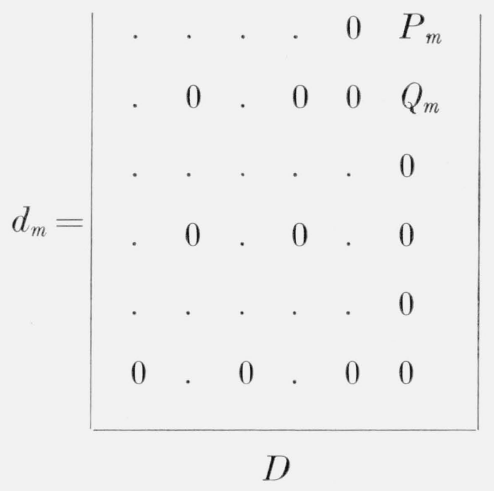

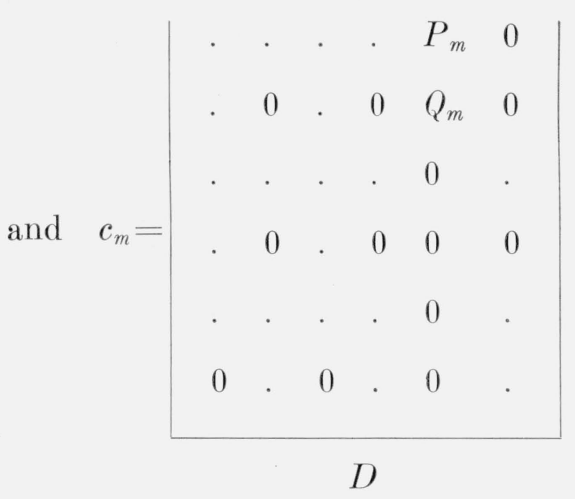

where the dots indicate generally finite factors obtained from the table. $D$ is the six by six determinant of the first six columns in table 1. Expanding these determinants leads to explicit, although lengthy, expressions for the fields.

\section{Far-Zone Fields}

The resulting integrals in region II are of the form

$$
I=-\frac{i}{2} \int_{-\infty}^{+\infty} F_{m}(h) H_{m}^{(2)}\left(u_{0} \rho\right) e^{-i h z} d h,
$$

where the dependence of the geometrical factors of the coated cylinder and the excitation parameters are lumped into $F_{m}(h)$. For large distances from the cylinder such that $k \rho \gg 1$, the Hankel and 
exponential functions are rapidly varying so the integral can be evaluated by the method of steepest descents. The procedure is straightforward [6] if it is remembered that the original contour in the $h$ plane must be indented above the branch point at $h=k$ and below the branch point at $h=-k$. The result is

$$
I \cong e^{i m \pi / 2} \frac{e^{-i k R}}{R} F_{m}\left(k_{0} \sin \theta\right),
$$

where $R=\left(\rho^{2}+z^{2}\right)^{1 / 2}$ and $\theta=\tan ^{-1}(\rho / z)$.

The far-zone form of the fields in region II are then simply obtained by replacing the Fourier operator $\Gamma$ by its steepest descent form, such that

$$
\Gamma F_{m}(h) \simeq 2 i \frac{e^{-i k R}}{R} \sum_{m=0}^{\infty} F_{m}\left(k_{0} \sin \theta\right) e^{i m \pi / 2} e^{-i m \phi},
$$

where terms which vary as $1 / R^{2}, 1 / R^{3}$, etc., are neglected.

\section{Equatorial-Plane Fields}

Even after making the far-zone approximation, the expressions for the fields are very cumbersome. The situation is simplified somewhat, however, if the observer is in the equatorial plane $(z=0$ or $\theta=$ $\pi / 2)$. This case is considered here and, furthermore, the slot is considered to be in the form of a narrow rectangle with its long side parallel to the cylinder axis. The integration, over the slot coordinates, indicated by eq (2) then simplifies to

$$
P_{m}(h) \simeq P_{m}(0) \simeq \frac{1}{4 \pi^{2} a} \int_{z_{1}}^{z_{2}} V\left(z^{\prime}\right) d z^{\prime},
$$

where the center line of the slot is at $\phi=0$ and $V\left(z^{\prime}\right)$ is the transverse voltage along the slot.

The far-field in the equatorial plane is then conveniently written

$$
E_{\phi}=\left[\frac{k_{0}}{2 \pi^{2}} \int_{z_{1}}^{z_{2}} V\left(z^{\prime}\right) d z^{\prime}\right] \frac{e^{-i k_{0 \rho}}}{\rho} P(\phi),
$$

where the pattern factor is given by

$$
P(\phi)=\frac{2 i}{\pi(k b)\left(k_{0} a\right)} \sum_{m=0}^{\infty} \frac{\epsilon_{m} \cos m \phi e^{i m \pi / 2}}{\left(\frac{k_{0} \mu}{k \mu_{0}}\right) H_{m}^{(2)}\left(k_{0} b\right) T_{m}-H_{m}^{(2),}\left(k_{0} b\right) L_{m}}
$$

with $\epsilon_{0}=1, \epsilon_{m}=2(m \neq 0)$ and

$$
\begin{aligned}
& T_{m}=J_{m}^{\prime}(k b) H_{m}^{(2) \prime}(k a)-J_{m}^{\prime}(k a) H_{m}^{(2) \prime}(k b), \\
& L_{m}=J_{m}(k b) H_{m}^{(2),}(k a)-J_{m}^{\prime}(k a) H_{m}^{(2)}(k b) .
\end{aligned}
$$

The prime over Bessel or Hankel function indicates a derivative with respect to its argument. As a partial check on this result it can be seen, if $b=a$ or if $\epsilon=\epsilon_{0}$ and $k=k_{0}$, that

$$
P(\phi)=\frac{1}{k_{0} a} \sum_{m=0}^{\infty} \frac{\boldsymbol{\epsilon}_{m} \cos m \phi e^{i m \pi / 2}}{H_{m}^{(2) \prime}\left(k_{0} a\right)},
$$

which is quite well known [1].

The equatorial-plane field is thus proportional to the integrated voltage moment along the slot, a simple radial factor that represents an outgoing spherical wave and a rather complicated azimuth factor. The structure of the solution is closely related to a two-dimensional (scalar) problem carried out previously [10] for an infinite axial slot with a uniform transverse voltage, $V$, throughout its length. The radiation field for this problem can be written

$$
E_{\phi}=-i 60 \epsilon_{\iota} \omega V\left(\frac{2}{\pi k_{0} \rho}\right)^{\frac{1}{2}} e^{-i\left(k_{0 \rho}-\pi / 4\right)} P(\phi),
$$

which has the form of an outgoing cylindrical wave with the same azimuthal dependence, $P(\phi)$, as for the finite slot. This two-dimensional counterpart is of further interest because it has a well defined acoustic analogy. In this instance, $E_{\phi}$ is proportional to the pressure field, in a medium whose wave number is $k_{0}$, emanating from a cylindrical radiator. The source is a rigid cylinder except for the narrow axial slot where the normal velocity is specified. Surrounding this cylinder is a film whose acoustic wave number is $k$ and a density, relative to the outer medium, equal to $\epsilon / \epsilon_{0}$.

Although the prime purpose, stated in the introduction, is to evaluate the effect of the dielectric coating, it does seem worthwhile for the sake of completeness to consider the effect when $\mu / \mu_{0}$ is different from unity. Composite materials can be produced whose macroscopic permeability differs from unity so the results may be quite significant in their own right.

Keeping in mind the above points, numerical computations of $P(\phi)$ were carried for two sizes of cylinders $\left(k_{0} a=2\right.$ and 3$)$ and for a range of values of $k_{0} b, \epsilon / \epsilon_{0}$, and $\mu / \mu_{0}$. Of course, a detailed study of the interrelation between these parameters would entail a great deal of numerical work. As a compromise, only a limited number of sets of calculations were carried out for the amplitude and phase of $P(\phi)$ for intervals of $\phi$ of 10 degrees, using available tables of Bessel functions [14]. Case I refers to the set of calculations where $\mu / \mu_{0}=1, \quad\left(\epsilon / \epsilon_{0}\right)^{\frac{1}{2}}=N$ and case II to the set where $\epsilon / \epsilon_{0}=1,\left(\mu / \mu_{0}\right)^{\frac{1}{2}}=M$. Setting $A=k_{0} a$ and $B=k_{0} b$, results were obtained for case I, taking

$$
\begin{aligned}
& A=2.0, N=2.0, \text { with } B=2.1,2.2,2.3 ; \\
& A=2.0, N=1.5, \text { with } B=2.2 ; \text { and } \\
& A=3.0, N=2.0, \text { with } B=3.2 .
\end{aligned}
$$

Corresponding values were obtained for case II with $M$ replacing $N$. For sake of comparison, the two relatively trivial situations, $A=B=2.0$ and $A=B=$ 3.0, were also considered.

The numerical results are summarized in tables 1,2 , and 3 with the appropriate values of $A, B$, and $N$ or $M$ at the head of each column of entries. If 
further values of $P(\phi)$ are required at smaller intervals of $\phi$, they can be computed directly from the following formula.

$$
P(\phi)=\frac{2}{\pi\left(k_{0} a\right)(k b)} \sum_{m=0}^{\infty} D_{m} \cos m \phi
$$

where $D_{m}$ is a Fourier coefficient listed in tables 4 and 6 for values of $m$ up to 10 . For the uncoated cylinder, where $A=B$, it is convenient to redefine the Fourier representation of $P(\phi)$ by

$$
P(\phi)=\frac{1}{\left(k_{0} a\right)} \sum_{m=0}^{\infty} d_{m} \cos m \phi,
$$

where $d_{m}$ is tabulated in table 7 .

The amplitude and phase ${ }^{3}$ of $P(\phi)$ are shown plotted in figures 2 to 7 . The set in figures 2 , a, and $2, b$, indicate, in a graphic way, the effect of varying the thickness of the dielectric coating for a fixed value of $a$. Figures $3, \mathrm{a}$, and $3, \mathrm{~b}$, show the influence of the dielectric constant of the coating material and figures 4 , a, and 4, b, pertain to a larger cylinder. It appears from these curves that the only significant change in the pattern, resulting from the addition of a dielectric coating, is to enhance the ripples in the curves. Physically, it may be supposed that the radiation from the slot travels around the periphery of the cylinder in both directions. The coating apparently "traps" these peripheral surface waves to some extent and consequently enhances the standing wave pattern. The fact that the period of this standing wave pattern does not depend essentially on the dielectric constant would indicate that surface wave is guided, with considerable leakage, along or just above the dielectric-air interface. The only essential effect of increasing the size of the cylinder is to increase the number of ripples and reduce their magnitude somewhat.

The set of curves in figures 5 to 7 correspond to a coating whose permeability relative to free space is $M^{2}$. The pronounced effect of wave trapping by a permeable layer is striking. The ripples in the pattern are much larger than the corresponding ones for the purely dielectric coating. Furthermore, it is apparent that the period of the ripples is modified by the permeability ratio $M^{2}$, indicating that the trapped peripheral surface waves are largely confined to within the film. Therefore, their phase velocity is mainly determined by the wave number $k$ rather than $k_{0}$.

\section{Concluding Remarks}

The analytical expressions developed herein are available for any future calculations of patterns of slotted cylinder antennas with dielectric coverings.

${ }^{3}$ Actually the quantity plotted is the phase lag, which is simply the negative of the phase.
The formulas could probably be programed for machine calculators if further numerical data are required. The complexity of the numerical procedures becomes excessive when the directions are not in the equatorial plane. It would seem to be desirable to search for an approximation technique to supplement this work. At the moment, the outlook is optimistic, using techniques based on simplified boundary conditions. In any event the rigorous calculations presented here should provide a comparative basis for checking any new approximate formulations.

On the basis of the limited calculations presented here it can be concluded that a thin dielectric film would have only a small effect on the radiation pattern for an axially slotted cylinder. On the other hand, a thin permeable film encasing the cylinder would substantially modify the pattern, indicating the presence of trapped peripheral surface waves.

We thank R. A. Hurd of the National Research Council of Canada for his comments and the verification of the final formulas in reference 10 .

\section{References}

[1] J. R. Wait, Radiation characteristics of axial slots on a conducting cylinder, Wireless Engr. 32, 316 (Dec. 1955).

[2] A. A. Pistolkors, Radiation from a transverse slit on the surface of a circular cylinder, J. Tech. Phys., U. S. S. R., 17, 377 (1947) (in Russian).

[3] George Sinclair, The pattern of slotted-cylinder antennas, Proc. Inst. Radio Engrs. 36, 1487 (1948).

[4] C. H. Papas and Ronold King, Currents on the surface of an infinite cylinder excited by an axial slot, Quart. Appl. Math. 16, 175 (1949).

[5] C. H. Papas, Radiation from a transverse slot on an infinite cylinder, J. Math. Phys. 28, 227 (1950).

[6] J. R. Wait, Radiation from a slot on a cylindrically tipped wedge, Can. J. Phys. 32, 714 (1954).

[7] S. Silver and W. K. Saunders, Field produced by a transverse slot on a circular cylinder, J. Appl. Phys. 21, 745 (1950).

[8] L. L. Bailin, Field produced by a slot on a large circular cylinder, Trans. Inst. Radio Engrs. (PGAP) AP-3, 128 (July 1955).

[9] J. R. Wait, and J. Kates, Radiation patterns of circumferential slots on moderately large conducting cylinders, Inst. Elect. Engrs. (London), Monograph No. $167 \mathrm{R}$, February 1956 (to be republished in Pt. C of the Proceedings)

[10] J. R. Wait Slotted-cylinder antenna with a dielectric coating, Addendum to Radio Physics Laboratory, Project Report 19-0-13 (Ottawa, Nov. 1954).

[11] D. G. Frood and J. R. Wait, An investigation of slot radiators in metal plates, Proc. Inst. Elec. Engrs. 103, 103 (Jan. 1956).

[12] A. W. Adey, Scattering of electromagnetic waves by co-axial cylinders, Can. J. Phys. 34, 510 (May 1956); also A. W. Adey (Ph. D. Thesis, Imperial College, Univ. of London, 1954).

[13] J. A. Stratton, Electromagnetic theory, p. 354 (McGrawHill Book Co., Inc., New York, N. Y., 1941).

[14] Scattering and radiation from circular cylinders and spheres, Math. Tables Proj. and M. I. T. Underwater Sound Laboratory, A. M. P. Report, 62-1-R (1945).

[15] R. S. Elliott, Spherical surface antennas, Trans. Inst. Radio Engrs. (PGAP) AP-4, 422 (July 1956). 
TABLe 1. List of coefficients

\begin{tabular}{|c|c|c|c|c|c|c|c|}
\hline$p$ & $a_{m p}$ & $b_{m p}$ & $A_{m p}$ & $B_{m p}$ & $c_{m p}$ & $d_{m p}$ & $x_{m p}$ \\
\hline 1 & $-\frac{m^{h}}{a} H_{m}(u a)$ & $i \mu \omega u H_{m}^{\prime}($ (иа) & $-\frac{m^{h}}{a} J_{m}(u a)$ & $i \mu \omega u J_{m}^{\prime}(u a)$ & 0 & 0 & $P_{m}$ \\
\hline 2 & $u^{2} H_{m}(u a)$ & 0 & $u^{2} J_{m}(u a)$ & 0 & 0 & 0 & $Q_{m}$ \\
\hline 3 & $-\frac{m \eta}{b} H_{m}(\cdot b)$ & $i \mu \omega \| I I_{m}^{\prime}(u h)$ & $-\frac{m^{b}}{b} J_{m}(u b)$ & $i \mu \omega u J_{m}^{\prime}(u h)$ & $\left.\frac{m^{\zeta}}{b} I_{m}\left(u_{0}\right\}\right)$ & $\left.-i \mu_{i} \boldsymbol{\omega} u_{0} H I_{m}^{\prime}\left(u_{0}^{\prime}\right)\right)$ & 0 \\
\hline 4 & $u^{2} I_{m}(u b)$ & 0 & $u^{2} J_{m}(u h)$ & 0 & $-u_{0}^{2} H_{m}\left(u_{0} 3\right)$ & 0 & 0 \\
\hline $5 \ldots$ & $-\frac{i k^{2}}{\mu \omega} u H_{m}(u b)$ & $-\frac{m^{3}}{h} I_{m}\left(u h_{1}\right)$ & $-\frac{i k^{2}}{\mu \omega} u J_{m}^{\prime}\left(u h_{3}\right)$ & $-\frac{m h}{b} J_{m}(u b)$ & $\left.+\frac{i k_{0}^{2} \iota_{0}}{\mu_{0} \omega} I I_{m}^{\prime}\left(u_{0}^{\prime}\right)\right)$ & $+\frac{m^{2}}{b} I_{m}\left(u_{0}^{\prime}\right)$ & 0 \\
\hline $6 \ldots$ & 0 & $u^{2} I I_{m}\left(u b^{\prime}\right)$ & 0 & $u^{2} J_{m}(u b)$ & 0 & $\left.-u_{0}^{2} H_{m}\left(u_{0}^{0}\right)\right)$ & 0 \\
\hline
\end{tabular}

TABLE 2. The pattern function, $P(\phi)$

Case I

\begin{tabular}{|c|c|c|c|c|c|}
\hline $\begin{array}{l}A \\
B \\
N \\
N\end{array}$ & \multirow[t]{2}{*}{$\begin{array}{l}2.0 \\
2.1 \\
2.0\end{array}$} & \multirow[t]{2}{*}{$\begin{array}{l}2.0 \\
2.2 \\
2.0\end{array}$} & \multirow[t]{2}{*}{$\begin{array}{l}2.0 \\
2.3 \\
2.0\end{array}$} & \multirow[t]{2}{*}{$\begin{array}{l}2.0 \\
2.2 \\
1.5\end{array}$} & \multirow[t]{2}{*}{$\begin{array}{l}3.0 \\
3.2 \\
2.0\end{array}$} \\
\hline$\phi$ & & & & & \\
\hline \multicolumn{6}{|c|}{ Amplitude $|P(\phi)|$} \\
\hline \multicolumn{6}{|l|}{ deg } \\
\hline 0 & 3.01542 & 3. 19057 & 3. 39700 & 3. 03693 & 3. 25498 \\
\hline 10 & 3. 01288 & 3. 19604 & 3. 40803 & 3.04000 & 3. 26369 \\
\hline 20 & 3. 00124 & 3. 20722 & 3. 43740 & 3.04148 & 3. 27721 \\
\hline 30 & 2. 96859 & 3. 20737 & 3. 46905 & 3. 02249 & 3. 25953 \\
\hline 40 & 2. 89763 & 3.16737 & 3. 46672 & 2. 96182 & 3. 16938 \\
\hline 50 & 2. 77345 & 3. 05397 & 3. 37882 & 2. 84294 & 3. 00552 \\
\hline 60 & 2. 59731 & 2.85054 & 3. 16491 & 2. 66134 & 2.84440 \\
\hline 70 & 2. 39951 & 2. 58432 & 2.83926 & 2. 43991 & 2. $7757 ?$ \\
\hline 80 & 2. 23553 & 2. 33949 & 2.50987 & 2. 24187 & 2. 74122 \\
\hline 90 & 2. 14398 & 2. 21216 & 2.34650 & 2. 13614 & 2.55112 \\
\hline 100 & 2. 09373 & 2. 19945 & 2. 39723 & 2. 10868 & 2. 13839 \\
\hline 110 & 1. 99247 & 2. 16949 & 2. 47621 & 2. 04517 & 1. 75636 \\
\hline 120 & 1. 75627 & 1. 97774 & 2.35044 & 1. 83122 & 1. 74087 \\
\hline 130 & 1. 36811 & 1. 57187 & 1. 91386 & 1. 43816 & 1. 82952 \\
\hline 140 & 0.919801 & 1. 04402 & 1. 24227 & 0.960739 & 1. 57059 \\
\hline 150 & .699471 & 0. 759246 & 0.758170 & .706317 & $0.918 \mathrm{C} 74$ \\
\hline 160 & .919829 & 1. 05545 & 1.12441 & .936848 & .575483 \\
\hline 170 & 1. 21183 & 1. 43974 & 1. 64774 & 1. 25289 & 1. 13915 \\
\hline 180 & 1. 32861 & 1. 59211 & 1.85397 & 1. 37960 & 1. 41882 \\
\hline \multicolumn{6}{|c|}{ Phase of $P(\phi)$} \\
\hline deg & deg & deg & deg & deg & deg \\
\hline 0 & 213.533 & 213. 044 & 211.124 & 212.873 & 268.001 \\
\hline 10 & 211.872 & 211. 274 & 209. 172 & 211. 238 & 265.428 \\
\hline 20 & 206. 967 & 206. 111 & 203.543 & 206. 379 & 257.900 \\
\hline 30 & 199. 001 & 197.917 & 194.805 & 198.410 & 245.748 \\
\hline 40 & 188.139 & 187.046 & 183.566 & 187.474 & 228.942 \\
\hline 50 & 174. 373 & 173.583 & 170.085 & 173.662 & 206.883 \\
\hline 60 & 157.447 & 157.142 & 153. 956 & 156. 842 & 179. 269 \\
\hline 70 & 137.032 & 136.872 & 133.942 & 136.562 & 148.124 \\
\hline 80 & 113.415 & 112.152 & 108.449 & 112.588 & 117.146 \\
\hline 90 & 88.386 & 84.477 & 78.257 & 86.450 & 87.094 \\
\hline 100 & 64. 621 & 57.919 & 49. 050 & 61.575 & 53. 961 \\
\hline 110 & 43. 553 & 35. 268 & 25.421 & 40.094 & 11.569 \\
\hline 120 & 24.517 & 15. 904 & 6.644 & 21. 221 & -33.607 \\
\hline 130 & 4. 689 & -3.355 & -10.683 & 1. 840 & -66.340 \\
\hline 140 & -23.597 & -30.693 & -34.167 & -25.805 & -89.882 \\
\hline 150 & -73.823 & -83.857 & -89.225 & -76.936 & -118.365 \\
\hline 160 & -116.591 & -129.818 & -145.407 & -122.107 & -201.661 \\
\hline 170 & -133.636 & -146.697 & -162.914 & -139.824 & -246.587 \\
\hline 180 & -138.047 & -150.933 & -166.999 & -144.379 & -253.828 \\
\hline
\end{tabular}

TABLE 3. The pattern function, $P(\phi)$ Case II

\begin{tabular}{|c|c|c|c|c|c|}
\hline $\begin{array}{l}A \\
B \\
M\end{array}$ & \multirow[t]{2}{*}{$\begin{array}{l}2.0 \\
2.1 \\
2.0\end{array}$} & \multirow[t]{2}{*}{$\begin{array}{l}2.0 \\
2.2 \\
2.0\end{array}$} & \multirow[t]{2}{*}{$\begin{array}{l}2.0 \\
2.3 \\
2.0\end{array}$} & \multirow[t]{2}{*}{$\begin{array}{l}2.0 \\
2.2 \\
1.5\end{array}$} & \multirow[t]{2}{*}{$\begin{array}{l}3.0 \\
3.2 \\
2.0\end{array}$} \\
\hline$\phi$ & & & & & \\
\hline \multicolumn{6}{|c|}{ Amplitude $|P(\phi)|$} \\
\hline deg & & & & & \\
\hline 0 & 3. 24416 & 2. 31378 & 2. 03562 & 2. 95396 & 2. 21707 \\
\hline 10 & 3,22684 & 2,39152 & 1,98875 & 2,96226 & 2,33487 \\
\hline 20 & 3,17033 & 2,60844 & 2,01230 & 2,97840 & 2,68975 \\
\hline 30 & 3,06456 & 2,90218 & 2,38631 & 297777 & 3,14516 \\
\hline 40 & 2,90306 & 3,15747 & 3,03508 & 2,92675 & 3,38660 \\
\hline 50 & 2. 69616 & 3. 23706 & 3. 60060 & 2. 79816 & 3. 15344 \\
\hline 60 & 2. 48507 & 3. 04068 & 3. 75866 & 2.59546 & 2. 55238 \\
\hline 70 & 2. 33989 & 2.58334 & 3. 33416 & 2. 37581 & 2.34847 \\
\hline 80 & 2. 31662 & 2. 10452 & 2. 39243 & 2. 24065 & 2. 93850 \\
\hline 90 & 2. 38889 & 2.07404 & 1. 53994 & 2. 24916 & 3. 32516 \\
\hline 100 & 2. 44442 & 2.54071 & 2. 05310 & 2. 32363 & 2. 81914 \\
\hline 110 & 2. 35724 & 2. 97488 & 3. 09308 & 2. 30334 & 1. 75203 \\
\hline 120 & 2. 07477 & 2. 98713 & 3. 59479 & 2. 06652 & 1. 93906 \\
\hline 130 & 1. 54389 & 2. 41705 & 3. 22720 & 1. 59490 & 2. 90073 \\
\hline 140 & 0.984364 & 1. 41820 & 2. 03580 & 1. 01666 & 2. 90070 \\
\hline 150 & .838475 & 0. 753540 & 0.563512 & 0.836482 & 1. 67907 \\
\hline 160 & 1. 25306 & $\begin{array}{l}\text { 1. } 63859 \\
\text { a }\end{array}$ & 1. 66202 & $\begin{array}{l}1.16270 \\
\text { 1. }\end{array}$ & 0.682360 \\
\hline 170 & 1. 66607 & 2.51597 & 2. 96109 & 1. 58455 & 2. 24128 \\
\hline 180 & 1. 82366 & 2.84481 & 3. 44712 & 1. 74717 & 2. 92864 \\
\hline
\end{tabular}

Phase $P(\phi)$

\begin{tabular}{r|c|c|c|c|c}
\hline deg & deg & deg & deg & deg & deg \\
0 & 195.746 & 181.927 & 203.376 & 198.928 & 244.215 \\
10 & 194.478 & 179.637 & 196.348 & 197.452 & 238.733 \\
20 & 190.593 & 173.720 & 176.260 & 193.110 & 226.331 \\
30 & 183.829 & 165.967 & 152.122 & 186.060 & 213.095 \\
40 & 173.816 & 157.381 & 134.364 & 176.340 & 200.028 \\
50 & 159.886 & 147.730 & 122.656 & 163.649 & 183.651 \\
60 & 141.448 & 135.613 & 113.362 & 147.238 & 155.984 \\
70 & 118.679 & 117.878 & 102.969 & 126.198 & 110.576 \\
80 & 93.6919 & 88.8039 & 85.1765 & 100.893 & 71.5498 \\
90 & 69.8430 & 49.4196 & 40.0331 & 74.6644 & 47.2343 \\
100 & 49.0554 & 18.1857 & -17.5717 & 51.5239 & 24.8248 \\
110 & 31.0754 & -0.997004 & -40.9840 & 32.4240 & -16.9857 \\
120 & 14.1408 & -14.2664 & -52.0521 & 15.7465 & -88.3266 \\
130 & -4.71149 & -26.4508 & -59.9543 & -1.96822 & -119.898 \\
140 & -36.3708 & -46.0996 & -69.9043 & -30.1175 & -134.839 \\
& & & & & \\
150 & -94.4545 & -120.536 & -125.229 & -68.1046 & -150.876 \\
160 & -132.229 & -174.999 & -221.830 & -130.191 & -262.113 \\
170 & -145.796 & -186.333 & -231.038 & -144.773 & -304.272 \\
180 & -149.319 & -188.884 & -232.834 & -148.435 & -308.423 \\
& & & & & \\
\hline & & & & & \\
\hline
\end{tabular}


TABLE 4. The pattern function, $P(\phi)$

\begin{tabular}{|c|c|c|c|c|c|}
\hline $\begin{array}{l}\mathrm{A} \\
\mathrm{B}\end{array}$ & \multirow[t]{2}{*}{$\begin{array}{l}2.0 \\
2.0\end{array}$} & \multirow[t]{2}{*}{$\begin{array}{l}3.0 \\
3.0\end{array}$} & $\begin{array}{l}\mathrm{A} \\
\mathrm{B}\end{array}$ & \multirow[t]{2}{*}{$\begin{array}{l}2.0 \\
2.0\end{array}$} & \multirow[t]{2}{*}{$\begin{array}{l}\text { 3. } 0 \\
\text { 3. } 0\end{array}$} \\
\hline$\phi$ & & & $\phi$ & & \\
\hline \multicolumn{3}{|c|}{ A mplitude $|P(\phi)|$} & \multicolumn{3}{|c|}{ Phase of $P(\phi)$} \\
\hline deg & & & deg & dey & deg \\
\hline 0 & 2. 91946 & 3. 01228 & 0 & 213.310 & 268.778 \\
\hline 10 & 2. 91276 & 3. 00388 & 10 & 211. 744 & 266.384 \\
\hline 20 & 2. 88927 & 2. 97364 & 20 & 207.088 & 259. 219 \\
\hline 30 & 2. 83980 & 2. 91104 & 30 & 199. 443 & 247. 294 \\
\hline 40 & 2. 75287 & 2. 81299 & 40 & 188.880 & 230.567 \\
\hline 50 & 2. 62188 & 2. 69702 & 50 & 175. 358 & 209. 146 \\
\hline 60 & 2. 45475 & 2. 59185 & 60 & 158. 719 & 183.822 \\
\hline 70 & 2. 27965 & 2. 49434 & 70 & 138.910 & 156. 249 \\
\hline 80 & 2. 13482 & 2. 34577 & 80 & 116.525 & 127.691 \\
\hline 90 & 2. 03659 & 2. 08708 & 90 & 93.2106 & 97.6070 \\
\hline 100 & 1. 95124 & 1. 76251 & 100 & 70.9499 & 63.3880 \\
\hline 110 & 1. 81096 & 1. 53456 & 110 & 50.6797 & 23. 9124 \\
\hline 120 & 1. 56192 & 1. 47497 & 120 & 31. 6970 & -14.0465 \\
\hline 130 & 1. 20474 & 1. 39522 & 130 & 11. 2645 & -43.9331 \\
\hline 140 & 0.827194 & 1. 10650 & 140 & -17.8471 & -68.8592 \\
\hline 150 & 659909 & 0.648455 & 150 & -65.1264 & -102.298 \\
\hline 160 & 830784 & 488877 & 160 & -104.451 & -176.508 \\
\hline 170 & 1. 05811 & .815190 & 170 & -121.274 & -216.288 \\
\hline 180 & 1. 14974 & .978586 & 180 & -125.766 & -224.045 \\
\hline
\end{tabular}

TABLE 5. Real and imaginary parts of $D_{m}$

Case I

\begin{tabular}{|c|c|c|c|c|c|c|c|c|c|c|}
\hline \multirow{2}{*}{$\frac{{ }_{m}^{\mathrm{A}}}{\mathrm{B}}$} & \multicolumn{2}{|c|}{$\begin{array}{l}2.0 \\
2.1 \\
2.0\end{array}$} & \multicolumn{2}{|c|}{$\begin{array}{l}2.0 \\
2.2 \\
2.0\end{array}$} & \multicolumn{2}{|c|}{$\begin{array}{l}2.0 \\
2.3 \\
2.0\end{array}$} & \multicolumn{2}{|c|}{$\begin{array}{l}2.0 \\
2.2 \\
1.5\end{array}$} & \multicolumn{2}{|c|}{$\begin{array}{l}3.0 \\
3.2 \\
2.0\end{array}$} \\
\hline & Real & Imaginary & Real & Imaginary & Real & Imaginary & Real & Imaginary & Real & Imaginary \\
\hline $\begin{array}{l}0 \\
1 \\
2 \\
3 \\
4\end{array}$ & $\begin{array}{l}-11.2226 \\
-23.5768 \\
-11.9489 \\
+4.2228 \\
+0.069988\end{array}$ & $\begin{array}{c}+2.09515 \\
-2.24456 \\
-22.4385 \\
-2.88595 \\
+3.61684\end{array}$ & $\begin{array}{l}-12.7066 \\
-25.8912 \\
-15.5227 \\
+7.9116 \\
+0.126520\end{array}$ & $\begin{array}{c}+1.96495 \\
-1.84874 \\
-23.7477 \\
-4.83094 \\
+4.56540\end{array}$ & $\begin{array}{l}-13.8062 \\
-29.1592 \\
-20.4830 \\
+22.2909 \\
+0.224247\end{array}$ & $\begin{array}{c}+2.95754 \\
-1.06811 \\
-24.2632 \\
-8.60814 \\
+5.78874\end{array}$ & $\begin{array}{l}-8.90168 \\
-18.7073 \\
-10.2028 \\
+11.8857 \\
+0.069761\end{array}$ & $\begin{array}{c}+1.68116 \\
-1.59443 \\
-17.3962 \\
-2.78592 \\
+3.01377\end{array}$ & $\begin{array}{l}-16.5595 \\
-28.0280 \\
-5.77076 \\
+44.6068 \\
+14.6735\end{array}$ & $\begin{array}{l}-15.6040 \\
-37.0156 \\
-49.4498 \\
-33.2523 \\
+39.6346\end{array}$ \\
\hline $\begin{array}{l}5 \\
6 \\
7 \\
8 \\
9\end{array}$ & $\begin{array}{l}-.728316 \\
+2.11 \times 10^{-8} \\
+0.018472 \\
-4.38 \times 10^{-9} \\
-0.000272\end{array}$ & $\begin{array}{c}+0.000862 \\
-.125133 \\
-6.57 \times 10^{-7} \\
+0.002381 \\
0\end{array}$ & $\begin{array}{l}-.909943 \\
-9.3 \times 10^{-7} \\
+0.022940 \\
-2.49 \times 10^{-8} \\
-0.000335\end{array}$ & $\begin{array}{l}+0.001481 \\
-.155779 \\
-2.48 \times 10^{-7} \\
+0.002948 \\
-1.15 \times 10^{-8}\end{array}$ & $\begin{array}{l}-1.11811 \\
-5.06 \times 10^{-5} \\
+0.027259 \\
-5.02 \times 10^{-8} \\
-0.000388\end{array}$ & $\begin{array}{l}+0.002667 \\
-.187316 \\
-7.99 \times 10^{-7} \\
+0.003456 \\
-7.4 \times 10^{-9}\end{array}$ & $\begin{array}{c}-.601487 \\
-2.2 \times 10^{-6} \\
+0.015030 \\
0 \\
-0.000217\end{array}$ & $\begin{array}{c}+0.000810 \\
-.010257 \\
-6.4 \times 10^{-7} \\
+0.001922 \\
0\end{array}$ & $\begin{array}{c}-13.0184 \\
-0.014469 \\
+.708769 \\
+8.4 \times 10^{-6} \\
-0.022283\end{array}$ & $\begin{array}{l}+0.665336 \\
-3.21999 \\
-0.000213 \\
+.137679 \\
-4.09 \times 10^{-5}\end{array}$ \\
\hline 10 & 0 & $-2.79 \times 10^{-5}$ & 0 & $-3.42 \times 10^{-5}$ & 0 & $-4.15 \times 10^{-5}$ & 0 & $-2.2 \times 10^{5}$ & $+2.7 \times 10^{--}$ & -0.003674 \\
\hline
\end{tabular}

TABle 6. Real and imaginary parts of $D_{m}$

Case II

\begin{tabular}{|c|c|c|c|c|c|c|c|c|c|c|}
\hline$m$ & Real & Imaginary & Real & Imaginary & Real & Imaginary & Real & Imaginary & Real & Imaginary \\
\hline $\begin{array}{l}0 \\
1 \\
2 \\
3 \\
4\end{array}$ & $\begin{array}{l}-10.1144 \\
-23.4645 \\
-20.9042 \\
+13.8792 \\
+0.071869\end{array}$ & $\begin{array}{r}+5.17046 \\
+4.80230 \\
-20.6570 \\
-4.47222 \\
+3.64883\end{array}$ & $\begin{array}{l}-8.52101 \\
-20.2125 \\
-27.0293 \\
+24.4545 \\
+0.141618\end{array}$ & $\begin{array}{c}+7.38167 \\
+10.7968 \\
-9.50909 \\
-14.3722 \\
+4.75266\end{array}$ & $\begin{array}{l}-6.04711 \\
-15.8423 \\
-22.7941 \\
+18.3439 \\
+0.292332\end{array}$ & $\begin{array}{c}+7.78317 \\
+13.1521 \\
-0.004936 \\
-38.8382 \\
+6.37791\end{array}$ & $\begin{array}{l}-8.06944 \\
-18.4190 \\
-14.1839 \\
+12.1601 \\
+0.52345\end{array}$ & $\begin{array}{l}+3.64159 \\
+2.76455 \\
-16.0117 \\
-2.99106 \\
+2.74666\end{array}$ & $\begin{array}{l}-18.5527 \\
-36.1706 \\
-27.5801 \\
+10.0303 \\
+59.0508\end{array}$ & $\begin{array}{l}-4.24158 \\
-14.4406 \\
-31.2589 \\
-51.4962 \\
+43.1818\end{array}$ \\
\hline $\begin{array}{l}5 \\
6 \\
7 \\
8 \\
9\end{array}$ & $\begin{array}{l}-.682888 \\
+.00000177 \\
+.015869 \\
-.0000000344 \\
-.0002201\end{array}$ & $\begin{array}{c}+0.000684 \\
-.111720 \\
-.00000034 \\
+.001981 \\
0\end{array}$ & $\begin{array}{l}-.816135 \\
-.0000011 \\
+.017896 \\
-3.51 \times 10^{-8} \\
-0.000242\end{array}$ & $\begin{array}{l}+0.001061 \\
-.128662 \\
-3.12 \times 10^{-7} \\
+0.002203 \\
-9.3 \times 10^{-9}\end{array}$ & $\begin{array}{l}-.975191 \\
-.000065 \\
+.020054 \\
-.000000037 \\
-.000266\end{array}$ & $\begin{array}{l}+0.001744 \\
-.146022 \\
-.00000064 \\
+.002436 \\
-.0000000053\end{array}$ & $\begin{array}{l}-.520690 \\
-.0000015 \\
+.012221 \\
0 \\
-0.000170\end{array}$ & $\begin{array}{c}+0.000487 \\
-.085703 \\
-.00000048 \\
+.001530 \\
0\end{array}$ & $\begin{array}{c}-16.4855 \\
-0.015533 \\
+.655667 \\
+.0000096 \\
-.019108\end{array}$ & $\begin{array}{l}+1.23400 \\
-3.30210 \\
-0.000158 \\
+.119019 \\
-.000045\end{array}$ \\
\hline 10 & 0 & -.000022 & 0 & -0.0000241 & 0 & -.000027 & 0 & -0.000017 & +.00000026 & -.002903 \\
\hline
\end{tabular}


TABLE 7. Real and imaginary parts of $d_{m}$

\begin{tabular}{|c|c|c|c|c|}
\hline \multirow{2}{*}{$m$} & \multicolumn{2}{|c|}{$\begin{array}{l}A=2.0 \\
B=2.0\end{array}$} & \multicolumn{2}{|c|}{$\begin{array}{l}A=3.0 \\
B=3.0\end{array}$} \\
\hline & Real & Imaginary & Real & Imaginary \\
\hline $\begin{array}{l}0 \\
1 \\
2 \\
3 \\
4\end{array}$ & $\begin{array}{l}-1.67622 \\
-3.50102 \\
-1.44185 \\
+1.82162 \\
+0.006261\end{array}$ & $\begin{array}{c}+0.310971 \\
-.400130 \\
-3.28618 \\
-0.270294 \\
+.454036\end{array}$ & $\begin{array}{l}-1.53852 \\
-2.54238 \\
-0.160813 \\
+4.33813 \\
+0.548382\end{array}$ & $\begin{array}{l}-1.47332 \\
-3.53029 \\
-4.62823 \\
-2.03120 \\
+2.81856\end{array}$ \\
\hline $\begin{array}{l}5 \\
6 \\
7 \\
8 \\
9\end{array}$ & $\begin{array}{c}-.090604 \\
0 \\
+0.002214 \\
0 \\
-0.000031\end{array}$ & $\begin{array}{c}+.000063 \\
-.015290 \\
0 \\
+0.000280 \\
0\end{array}$ & $\begin{array}{r}-.884368 \\
-.000506 \\
+.048952 \\
0 \\
-0.001603\end{array}$ & $\begin{array}{r}+0.023621 \\
-.223038 \\
-.000008 \\
+.009409 \\
0\end{array}$ \\
\hline 10 & 0 & -0.000003 & 0 & -0.000245 \\
\hline
\end{tabular}

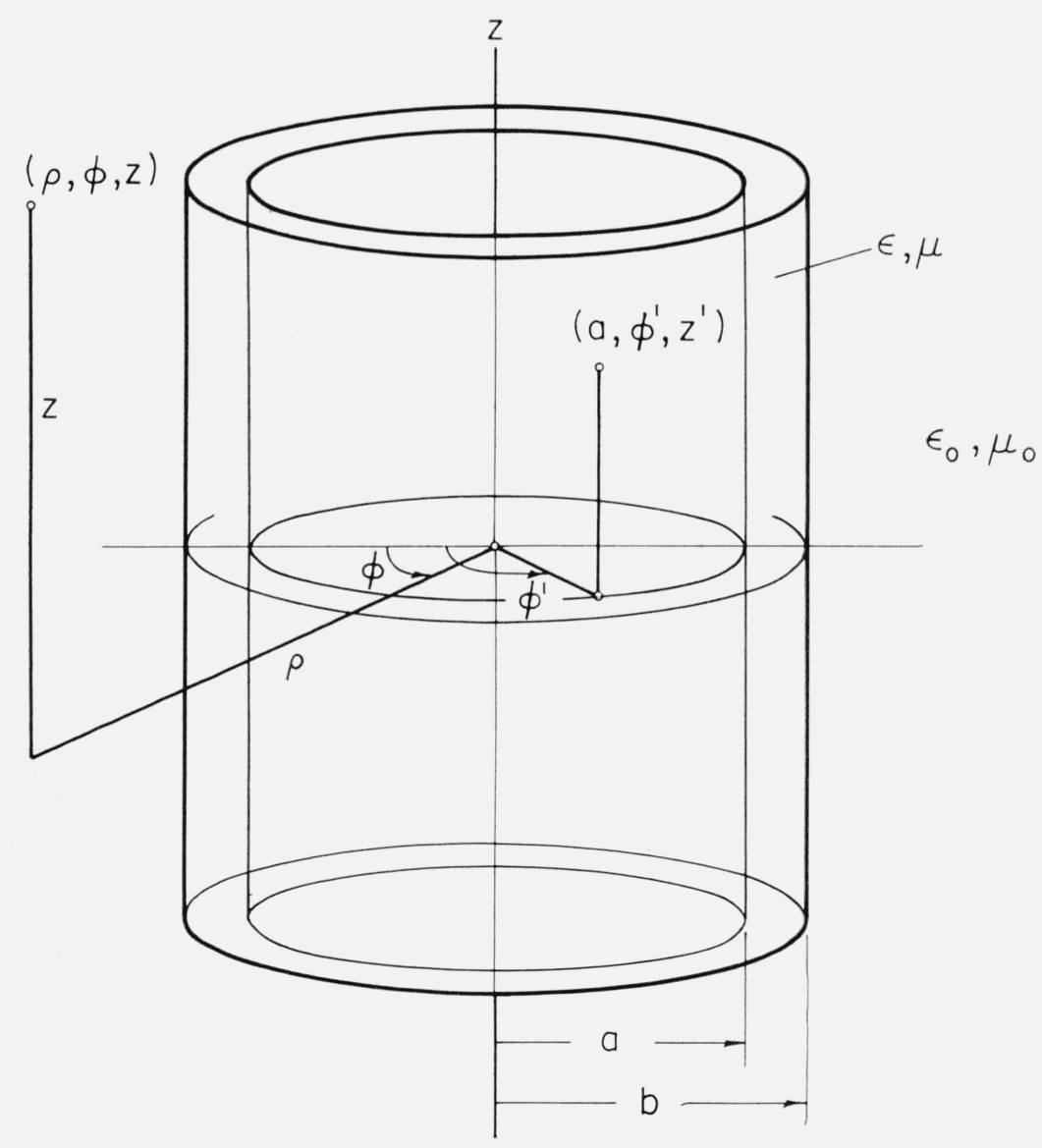

Figure 1. Coordinate systems for the dielectric-coated cylinder of infinite length. 

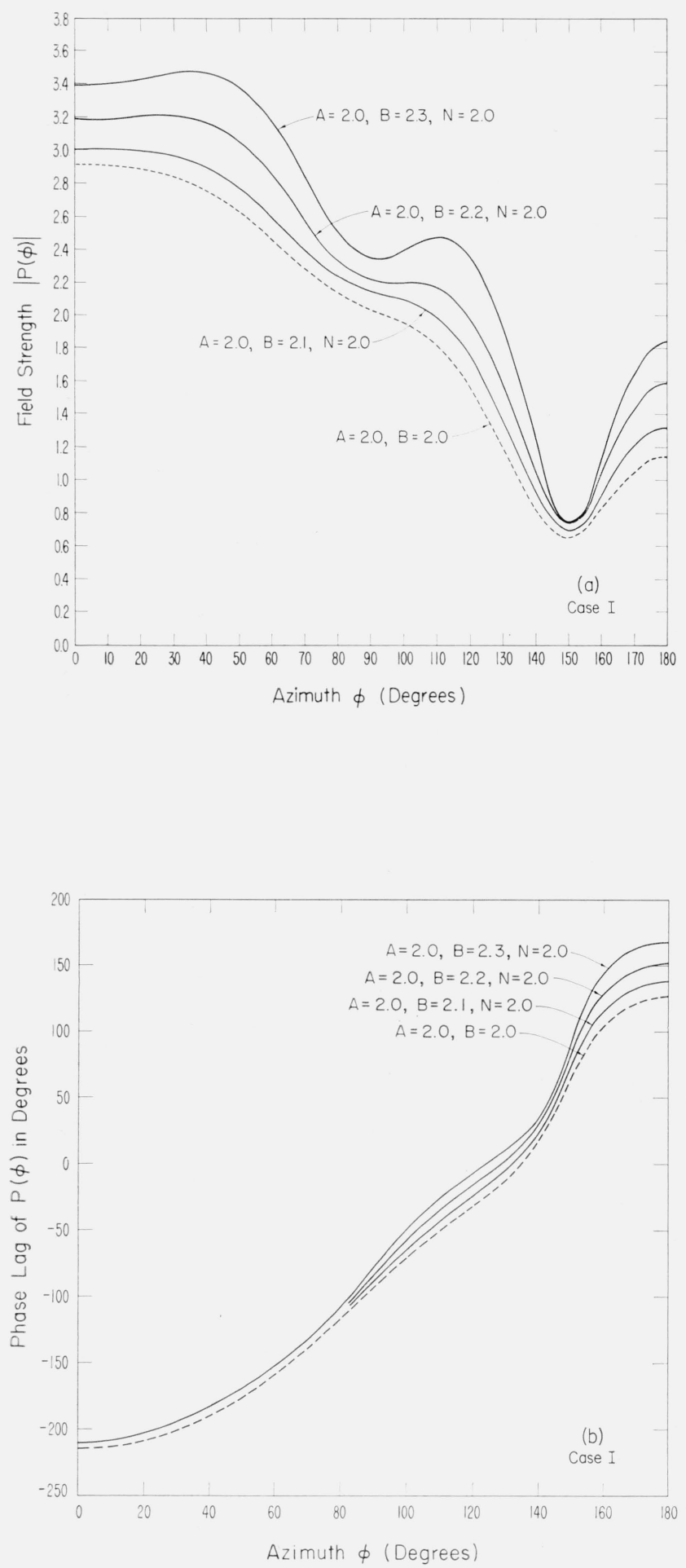

Figure 2. Pattern of slotted-cylinder antenna with a dielectric coating showing effect of thickness.
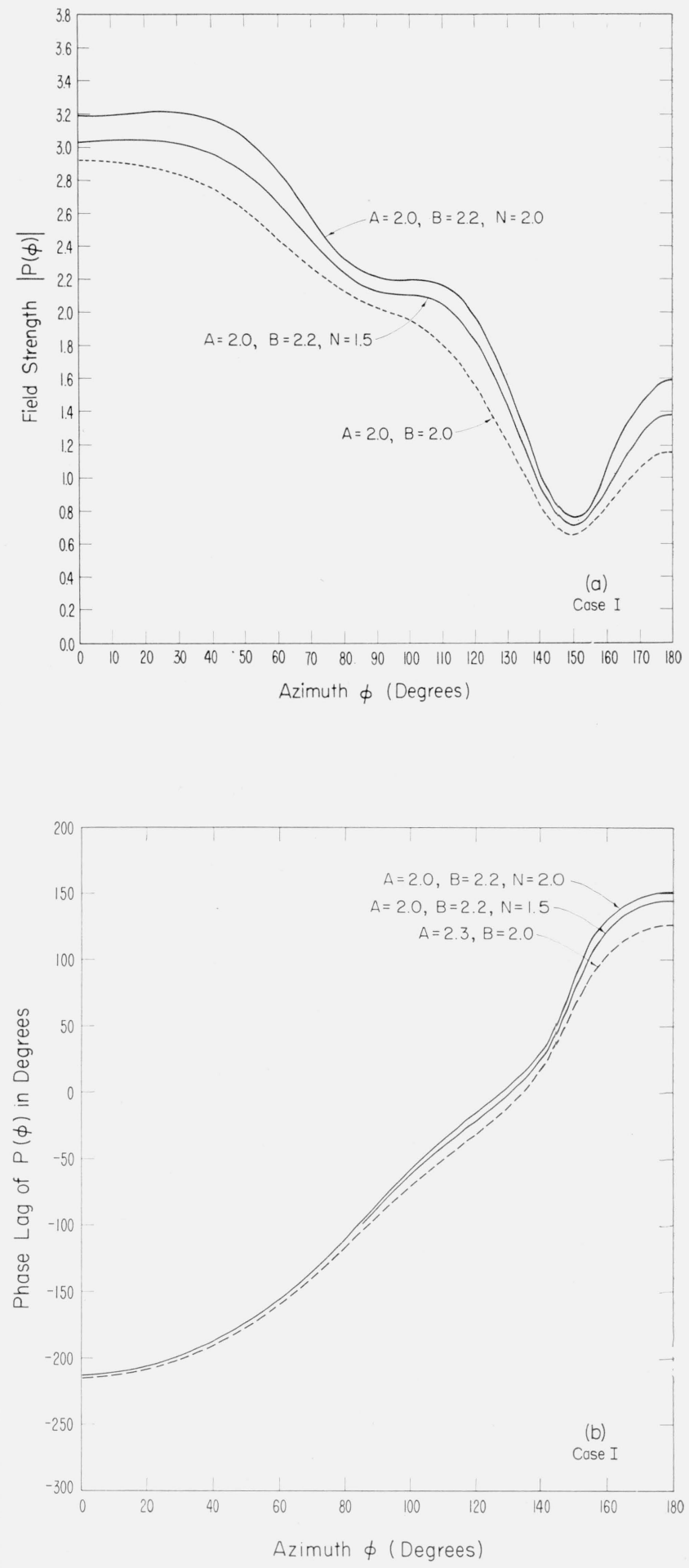

Figure 3. Pattern of slotted-cylinder antenna with a dielectric coating showing effect of refractive index. 

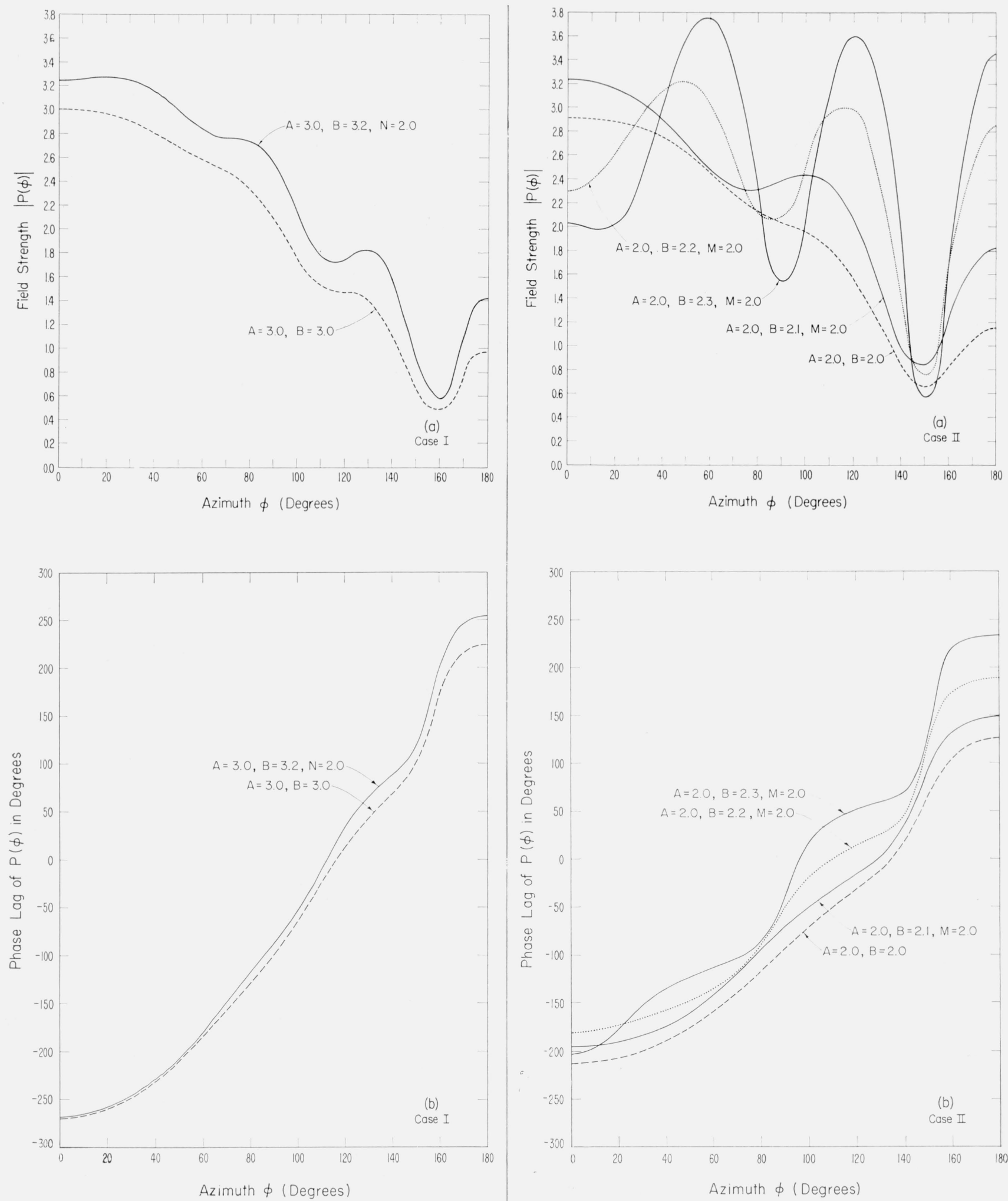

Figure 4. Pattern of slotted-cylinder antenna with a dielectric coating for a larger cylinder.

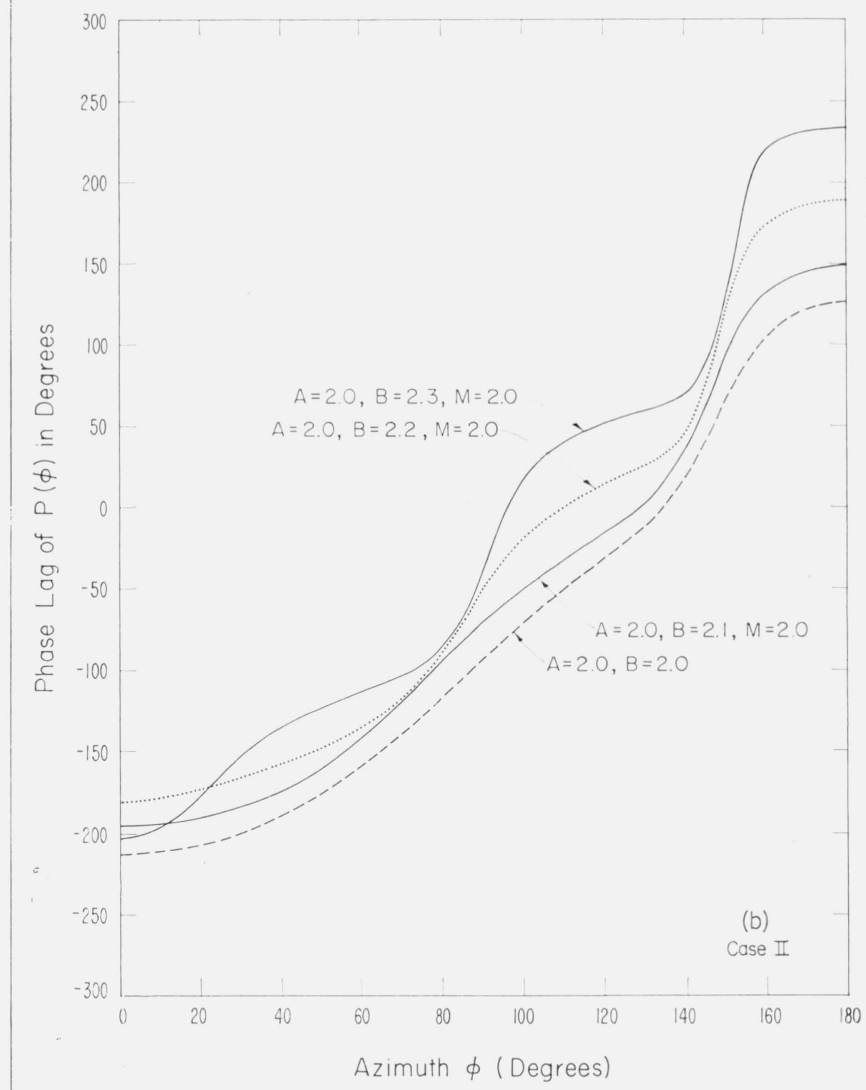

Figure 5. Pattern of slotted-cylinder antenna with a permeable coating showing effect of coating thickness. 

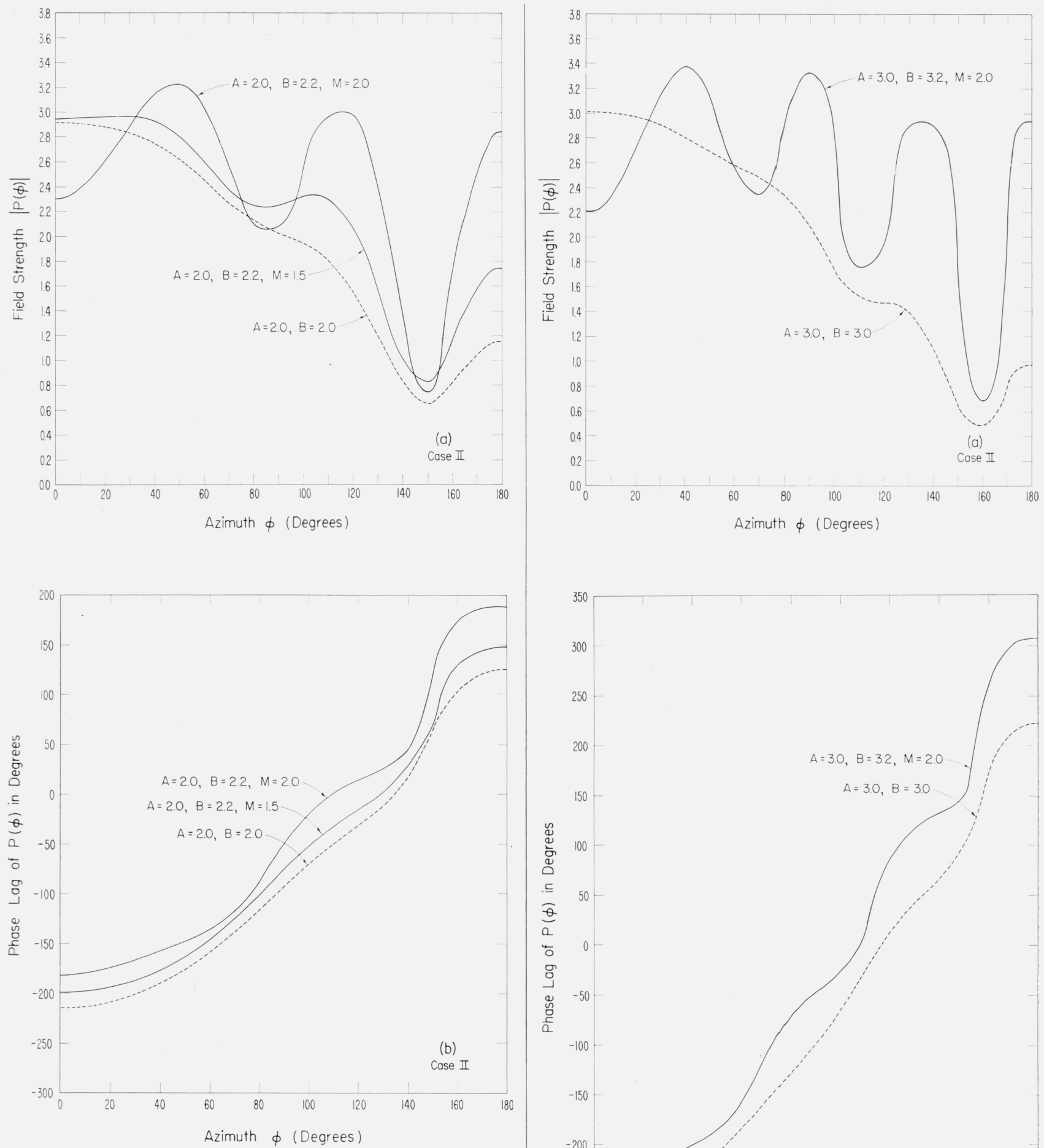

Figure 6. Pattern of slotted-cylinder antenna with a permeable coating showing effect of refractive index.

Boulder, Colo., October 25, 1956.

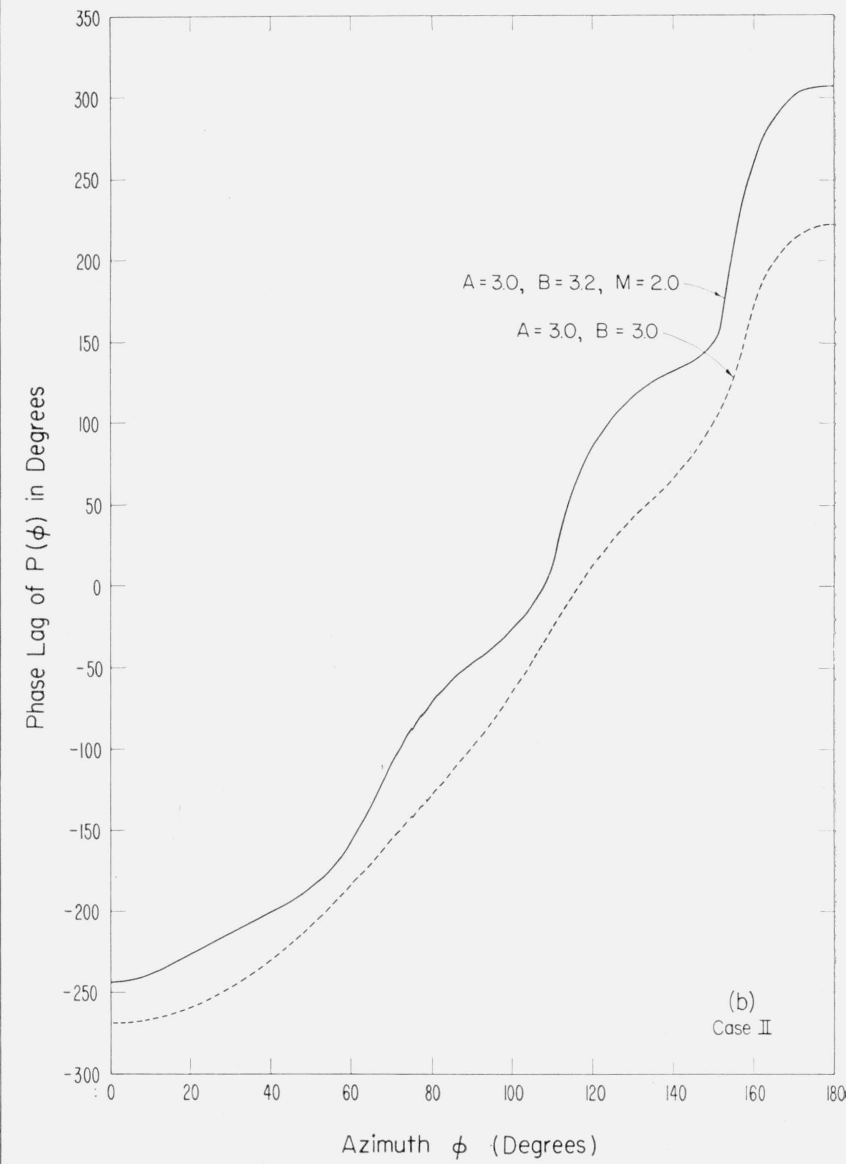

Figure 7. Pattern of slotted-cylinder antenna with a permeable coating for a larger cylinder. 